\section{Practice Patterns in the Management of Myasthenia Gravis: A Cross-Sectional Survey of Neurologists in the United States}

Vera Bril, $\mathrm{MD}^{1}$; Jacqueline Palace, $\mathrm{MD}^{2}$; Tahseen Mozaffar, $\mathrm{MD}^{3}$; Deborah Gelinas, $\mathrm{MD}^{4}$; Edward Brauer, PharmD ${ }^{4}$; Paul Nisbet, $\mathrm{PhD}^{5}$; Gil I. Wolfe, $\mathrm{MD}^{6}$

${ }^{1}$ Toronto General Hospital, University Health Network, University of Toronto, Toronto, Canada

${ }^{2}$ Department of Clinical Neurology, John Radcliffe Hospital, Oxford University Hospitals Trust, Oxford, UK

${ }^{3}$ UC Irvine-MDA ALS and Neuromuscular Center, University of California, Irvine, California, USA

${ }^{4}$ Medical Affairs, argenx, Boston, Massachusetts, USA

${ }^{5}$ One Research, LLC, Charleston, South Carolina, USA

${ }^{6}$ Department of Neurology, University at Buffalo Jacobs School of Medicine and Biomedical Sciences, State University of New York, Buffalo, New York, USA

\section{ABSTRACT}

Background: Management of myasthenia gravis (MG), a rare immunoglobulin $\mathrm{G}$ autoantibody-mediated neuromuscular junction disorder, is driven by physician experience. To gain insight into current practices and physician needs, neurologists' use of guidelines and disease activity evaluations to manage MG was assessed.

Methods: In November and December of 2020, a quantitative, cross-sectional, 51-item, online surveybased study was used to collect data from 100 community neurologists, from 31 US states, who treat MG. Differences across ratio variables were analyzed via Chi-square and $t$ tests, at a significance level of $P<0.05$.

Results: Of respondents, $76 \%$ reported using clinical judgment rather than guidelines to inform treatment decisions, and only $29 \%$ reported awareness of the updated 2020 International Consensus Guidance for Management of Myasthenia Gravis. Treatment patterns reported include use of prednisone-equivalent corticosteroid doses $\leq 10 \mathrm{mg} /$ day for $\geq 6$ months (76\% of respondents). When corticosteroids are contraindicated or after failure of an initial nonsteroidal immunosuppressant therapy (NSIST), immunoglobulin therapy is the respondents' preferred initial treatment in patients with acetylcholine receptor antibody-positive generalized MG (vs a second NSIST). Respondents expressed interest in more guidance on crisis management, initiating/titrating maintenance medications, and managing patients with comorbidities.

Conclusions: Respondents to this survey reported varied approaches to MG management and, in some clinical settings, heavier reliance on clinical judgment than on available consensus-based guidance. Also observed was potential underutilization of NSISTs in patients for whom corticosteroids are contraindicated, with reliance, instead, on immunoglobulin.

Keywords: cross-sectional survey, myasthenia gravis, clinical practice guideline, clinical practice patterns, disease management

\section{Introduction}

Myasthenia gravis $(\mathrm{MG})$ is a rare acquired autoimmune disease, characterized by fluctuating muscle weakness $(1,2)$ frequently affecting facial, bulbar, neck, respiratory, and limb muscles (2). MG results from abnormal binding of pathogenic autoantibodies to components of the neuromuscular junction (NMJ), disrupting normal neuromuscular transmission and leading to variable muscle weakness that typically worsens with exertion (1). MG pathophysiology is primarily centered on acetylcholine receptors (AChRs). Up to 85\% of patients with generalized MG (gMG) have immunoglobulin G (IgG) autoantibodies (3), which have a direct effect on skeletal AChRs, inducing muscle weakness (1). A small proportion of patients have MG involving antibodies to muscle-specific tyrosine kinase (MuSK) or lipoprotein receptor-related protein 4 (LRP4) (1). MG is the most common acquired NMJ disorder, according to data published from 1990 to 2014, with an annual international prevalence ranging from 5.35 to 35 per 100,000 persons and an annual international incidence ranging from 0.3 to 2.8 per 100,000 persons $(1,4)$.

There are broadly accepted therapies for $\mathrm{MG}$, although often with low levels of evidence to support their use (5) and, until recently, there were no international recommendations to guide care for MG. As treatment options continued to expand, improved strategies for managing this heterogeneous disease became necessary (6). Accordingly, a 15-member international task force was convened in 2013 by the Myasthenia Gravis Foundation of America (MGFA) to address this unmet need. The task force published its International Consensus Guidance for Management of Myasthenia Gravis in 2016 (7), covering symptomatic and immunosuppressive treatment, therapeutic plasma exchange (TPE) and intravenous 
immunoglobulin (IVIg), management of myasthenic crisis, thymectomy, juvenile MG, MuSK MG, and MG in pregnancy (7). The panel was reconvened in 2019, adding a new member representing South America, to review and update the 2016 recommendations and guidance. The revised International Consensus Guidance was published in November 2020, with new recommendations for use of rituximab, eculizumab, and methotrexate; management of immune checkpoint inhibitor (ICI)-induced MG; and early immunosuppression in ocular MG (8).

Management of MG focuses on reducing symptoms with an acetylcholinesterase inhibitor (AChEi) or modulating the immune system (9). Pyridostigmine is the preferred first-line AChEi for MG (2). In early or mild MG, pyridostigmine can provide rapid and substantial improvement in muscle strength; however, treatmentrelated adverse effects (AEs) are common and therapeutic benefit is often limited (2). Most patients with MG will require immunomodulatory treatment to achieve treatment goals and restore physical activity (1, 2). Oral corticosteroids are the primary immunomodulatory therapy for maintenance management of MG (2). Clinical benefit is relatively fast, with most patients experiencing improvement within weeks (10). At high doses or with longterm use, however, corticosteroids can cause significant AEs, including steroid-induced diabetes, hypertension, cataracts, glaucoma, and neuropsychiatric disturbances (9, 10).

Azathioprine is a nonsteroidal immunosuppressive therapy (NSIST) for MG, with a relatively long latency to clinical effectiveness, estimated as 6 to 18 months (10). Use of an NSIST in gMG can increase risk for serious infection and slightly increases incidence of some cancers, including squamous cell carcinoma and lymphoma (9). TPE and IVIg are rapid and effective immunomodulating treatments often used for acute myasthenic exacerbation or crisis; however, treatment effect is not durable beyond a matter of weeks (10) and this option is not available to some patients because of treatment cost or lack of necessary equipment. New and emerging classes of pharmacologic agents being used in MG target B cells, T cells, complement, and the neonatal FC receptor (9). There is hope that newer therapies will better address unmet needs in the management of MG.

While often effective, older immunosuppressive therapies can require trials of up to 12 months to determine efficacy. The increase in therapeutic options, particularly progress in targeted immunotherapies, holds promise for safer, faster, and more sustained benefit in patients with MG (11) and for patients whose disease is refractory to or who are intolerant of standard therapies $(12,13)$. However, randomized clinical trial data that guide MG management remain limited and often cannotbe applied across the various subpopulations of MG because of restrictive trial entry criteria. Consensus-driven treatment recommendations that are updated to reflect therapeutic advances are helpful in this environment, but their dissemination and adoption among MG health care providers has not been studied.

This quantitative cross-sectional online survey was designed to gain a better understanding of US community neurologists' standard treatment practices in MG and the extent of formal treatment guidance utilization. We assessed how community neurologists use available treatment guidance and survey instruments to measure disease activity to manage patients with MG. We also investigated whether the 2020 International Consensus Guidance had yet impacted clinical practice, examined potential barriers preventing its use, and explored areas where additional, targeted, education may be indicated.

\section{Materials and Methods}

The study was quantitative and utilized a crosssectional design to collect survey-based data from community neurologists in the United States. The 10-minute online survey was constructed to investigate patient assessment methods, as well as treatment decisionmaking, among neurologists who manage patients with MG. All survey questions were developed by and finalized via discussion-based input from all authors. Prior to online study launch, pilot testing of 2 community neurologists was used to validate appropriate wording of questions, comprehensiveness of response sets, and inclusion of clinically salient topics/questions. The pilot test participants self-administered the online survey while concurrently participating in a telephone interview with the study project lead. Responses from pilot testing were not included in final survey data analyses.

The 5l-item, self-administered questionnaire (Appendix A) was designed to elicit information from practicing community neurologists on a variety of $\mathrm{MG}^{-}$ related topics, including familiarity with and usage of the International Consensus Guidance for clinical decisionmaking; treatment goals (full treatment goal definitions are in Appendix B); clinical features and evaluations or measures commonly used to diagnose, track patient progress, or guide clinical decisions; typical induction and maintenance corticosteroid dosages; outcome measures used to determine response to therapy; and physician approaches to patient education, especially regarding thymectomy and expectations for treatment.

The quantitative survey data were collected between November 19, 2020, and December 3, 2020. A multifaceted approach to ensure the quality of data and collection 
Table 1. Demographic information for online survey respondents (community neurologists)

\begin{tabular}{|c|c|}
\hline Respondent characteristics & $\mathrm{N}=100$ \\
\hline \multicolumn{2}{|l|}{ Number of patients with MG treated per year } \\
\hline Mean (SD) & $66(104.6)$ \\
\hline Median & 28 \\
\hline Range & $3-500^{\mathrm{a}}$ \\
\hline \multicolumn{2}{|l|}{ Primary practice setting, $\%$} \\
\hline Not university affiliated & 51 \\
\hline University affiliated & 49 \\
\hline \multicolumn{2}{|l|}{ Board certifications (may be $\geq 1$ per respondent), $\%$} \\
\hline Neurology & 89 \\
\hline Electrodiagnostic medicine & 37 \\
\hline Neuromuscular medicine & 37 \\
\hline Pediatric neurology & 11 \\
\hline \multicolumn{2}{|l|}{ Primary practice type, $\%$} \\
\hline Solo & 17 \\
\hline Single-specialty partnership or group ( $\geq 2$ physicians) & 28 \\
\hline Multi-specialty partnership or group ( $\geq 2$ physicians) & 55 \\
\hline \multicolumn{2}{|l|}{ Number of years since residency/training } \\
\hline Mean (SD) & $16(9.9)$ \\
\hline Median & 15 \\
\hline Range & $3-59$ \\
\hline
\end{tabular}

Abbreviations: MG, myasthenia gravis; SD, standard deviation.

${ }^{a}$ Of respondents, 1 reported seeing 300 patients; 3 reported seeing 400 patients; 1 reported seeing 450 patients; and 2 reported seeing 500 patients.

methods was used. A random sample of 1300 confirmed community neurologists were recruited from e-Rewards, a Dynata, LLC (Shelton, Connecticut) subsidiary performing health care market research; panel members who met eligibility criteria and completed the survey were compensated for their time and opinions. Prospective USbased survey respondents were already members of the Dynata physician panel, having completed the registration and enrollment process, including provision of medical education number to confirm physician status. Dynata monitors its panel pool to ensure members do not claim different specialties for different surveys and that only members of the targeted specialty are included in the email invitations, which also minimizes screen failures. Secondlevel verification for potential fraudulent responders was accomplished via checks for duplicate participants, using variables such as IP address, matching across demographic data points, and checks of device-related data via thirdparty digital fingerprint technology.

Qualifications for survey participation included specialization in neurology, having been in practice for at least 2 years since residency completion, treating or consulting on at least 2 patients with MG per year, and practicing in the United States (physicians practicing in the state of Vermont were excluded due to legal restrictions regarding online survey participation). The email invitation provided a general description of the survey topic (ie, "neurology patient management") and a link to the online survey. To prevent respondents from taking the survey more than once, each email invitation was linked to a unique identifier. Survey respondents remained anonymous to the study sponsor and the authors of this manuscript. No patient data were obtained and no questions were asked of physician respondents that would allow identification of any patients. For these reasons, this study was exempt from institutional review board (IRB) approval under United States Code of Federal Regulations Title 45 Part 46.101(b) (2); the study received a formal letter of exemption from the Copernicus Group IRB (Cary, North Carolina).

Data quality was monitored using a variety of strategies. Final data were reviewed to ensure that answers were given consideration and were not simply randomly generated responses. Logic checks, which were built into the survey script, were used to monitor and detect responses that were potentially illogical or inconsistent. Participants $(n=2)$ whose data indicated overuse of non-responses (ie, "NA") were removed from the sample before final analysis, as was 1 participant who completed all responses in less than $30 \%$ of the median duration to complete the survey. Each respondent's route through the survey was checked to 
ensure the respondent did not bypass any significant section. Descriptive statistics were performed using Chi-square and $t$ tests to evaluate differences across ratio variables, including board certification subgroup, practice setting, and years in practice. Statistical significance was assessed at the alpha level of $P<0.05$. Descriptive analyses were performed using SPSS Statistics 26.0 (IBM; Armonk, New York), and data analysis was performed by study author PN.

\section{Results}

Demographics for the 100 community neurologists who satisfied selection criteria and successfully completed the survey are described in Table 1. Thirty-one of the 50 US states were represented in the survey sample (Figure 1). Of respondents, $51 \%$ are affiliated with a university. The majority (89\%) are board certified (BC) in neurology, 37\% in electrodiagnostic medicine/clinical neurophysiology, $37 \%$ in neuromuscular medicine (NM), and $11 \%$ in pediatric neurology. University-affiliated neurologists were significantly more likely to report being $\mathrm{BC}$ in other domains, particularly in NM. The greatest differences in practice patterns were observed between neurologists who are board certified in neuromuscular medicine (NMBC) and neurologists who are non-NMBC. Importantly, there was a large difference between these 2 groups in reported awareness of the 2020 International Consensus Guidance: only $19 \%$ of the 63 non-NMBC respondents were aware of the newly released International Consensus Guidance vs almost half (46\%) of the $37 \mathrm{NMBC}$ respondents.

Overall, most respondents (76\%) reported using clinical judgment rather than treatment guidelines to make MG treatment decisions (Figure 2A). Decisionmaking processes were most often reported as guided by assessments of patient strength, activities of daily living (ADLs), and tolerance of treatment rather than by any single recommended goal, such as the 2016 International Consensus Guidance treatment goal of minimal manifestation status (MMS). Only $44 \%$ of respondents reported using MMS. The majority (64\%) of respondents reported not using any specific guideline for MG treatment decisions.

Of respondents who did report use of a guideline to manage treatment $(n=36$; Figure $\mathbf{3 A}$ ), more than half reported using "American Academy of Neurology" (AAN); others reported "MGFA guidelines" or "other." Respondents were asked to select any clinical treatment guidelines they use for clinical management of patients with MG; those data, as well as verbatim responses for "other" clinical tools, are in Figure 3B.

In response to a survey question inquiring whether they would offer thymectomy to patients with purely ocular MG,
$68 \%$ of NMBC neurologists replied affirmatively compared to $43 \%$ of non-NMBC neurologists. For patients with gMG with a positive AChR antibody status, $95 \%$ of both $\mathrm{NMBC}$ and non-NMBC neurologists indicated that they would offer thymectomy. In contrast, only $62 \%$ of NMBC neurologists would offer thymectomy to patients with a positive MuSK antibody status, compared to $78 \%$ of nonNMBC neurologists.

The diagnostic tests reported as most frequently used by all survey respondents (both NMBC and non-NMBC) to confirm diagnosis of MG were AChR antibody panel (87\%), MuSK antibodies (77\%), and repetitive nerve stimulation (RNS; 75\%). Single-fiber electromyography (SFEMG) was reported by $46 \%$ and AChEi challenge and LRP4 antibody by $37 \%$; however, LRP4 antibodies are also used in some cases to support diagnosis. Other diagnostic tests included ice pack, which was reported as being used by $31 \%$, and “other," by $1 \%$. In seronegative patients, respondents said they confirm MG by RNS (78\%), SFEMG (63\%), AChEi challenge (45\%), and "other" (3\%). Approximately a third (31\%) of all respondents reported monitoring autoantibody levels to evaluate response to therapy. Those reporting use of autoantibody levels to assess treatment response did not differ by practice setting or BC status. Of respondents who reported using autoantibody testing, the type utilized most often is AChR serologies. Further measures used to track MG disease status are summarized in Figure 2B.

The majority (74\%) of respondents reported targeting a prednisone-equivalent corticosteroid dose $\leq 10 \mathrm{mg}$ /day for chronic use ( $\geq 6$-month periods); however, when asked the percentage of patients with gMG in whom the respondents avoided corticosteroids, the mean percentage was $27 \%$ for

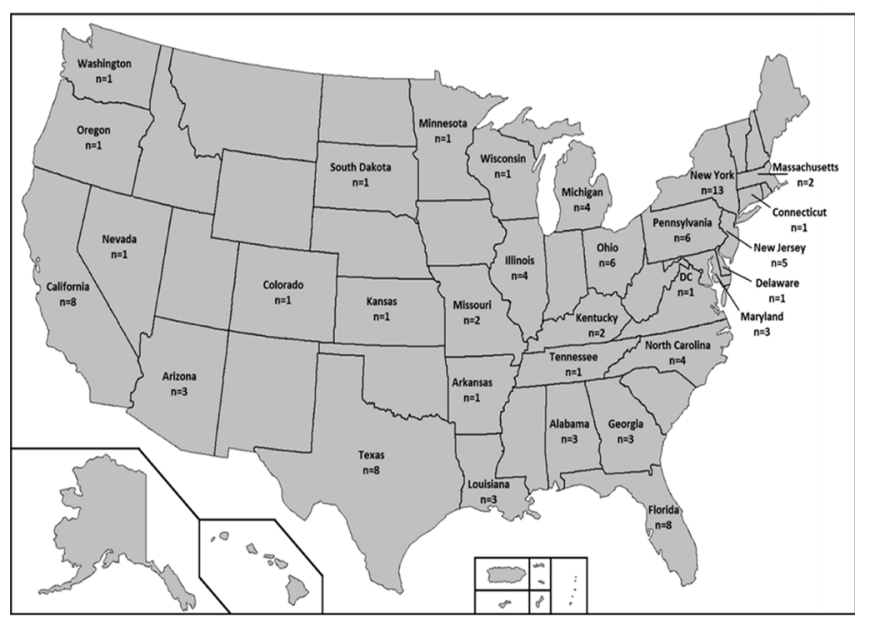

Figure 1. Number of Survey Respondents, by State

Map of the United States, with number of survey respondents from each state listed as n value. The survey email invitation provided only a general description of the survey topic (i.e., "Neurology Patient Management") and a link to access the online survey. $\mathrm{N}=100$. 


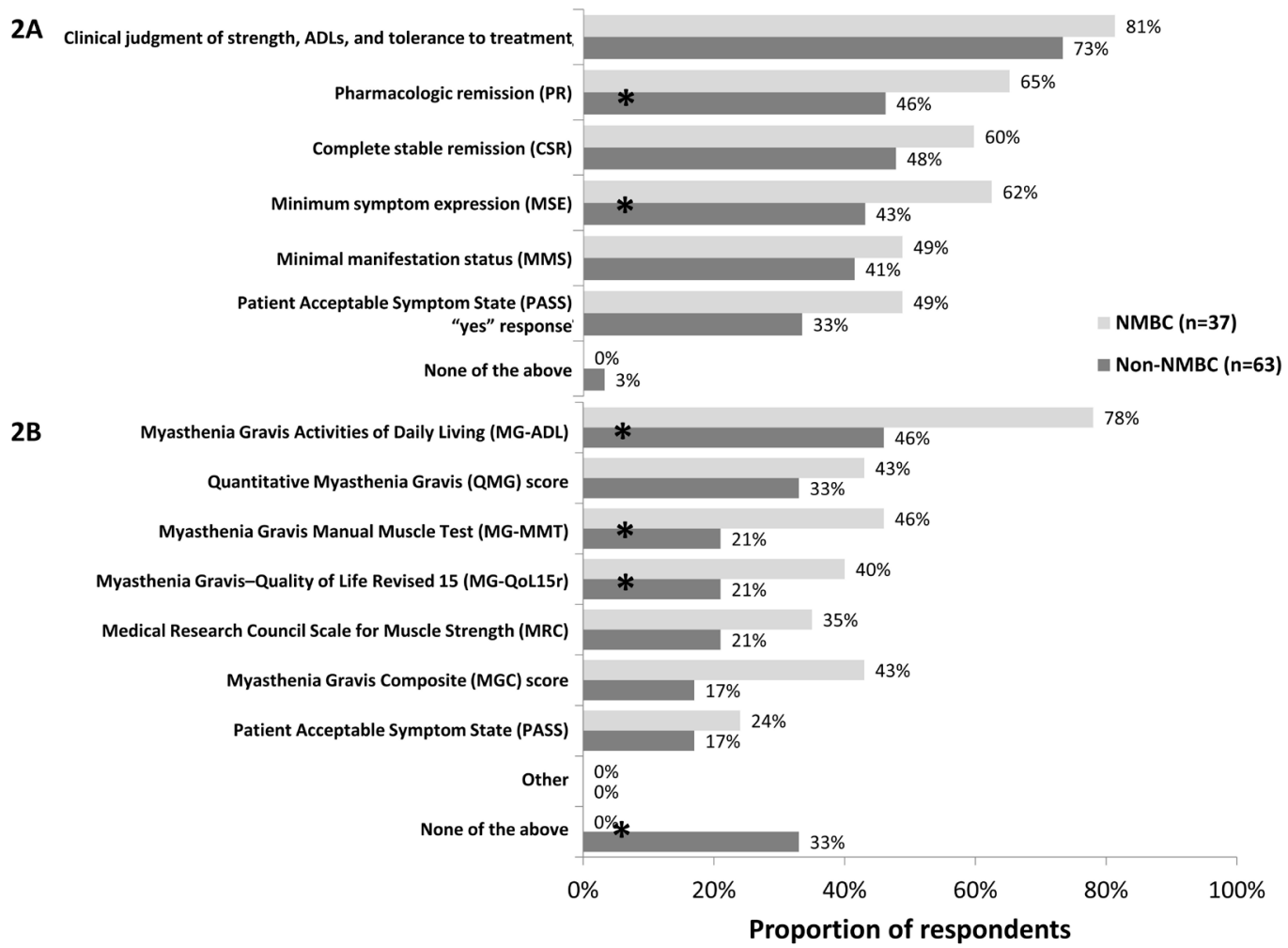

Figure 2. Goals and Assessment Measures Used for MG Treatment Decisions

Abbreviations: ADL, activity of daily living; gMG, generalized myasthenia gravis; MG, myasthenia gravis; NMBC, neuromuscular medicine board certified.

This survey focused on gMG unless otherwise specified. Data allowed selection of more than 1 measure where applicable, so percentages sum $>100 \%$.

Survey question 2A: "Which disease-specific measures do you use routinely in your clinic to track MG status?"; 2B: "Which of the following treatment goal(s) do you use to guide MG treatment decisions?”. (N=100).

*Indicates difference was statistically significant $(P<0.05)$.
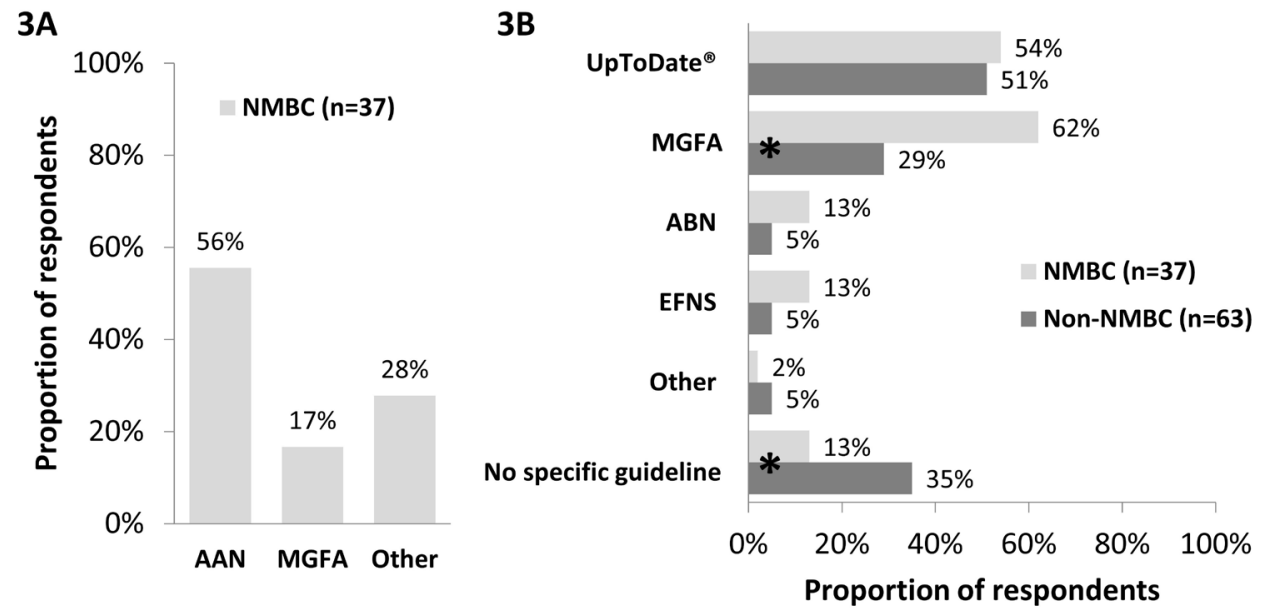

Figure 3. Use of Treatment Guidelines in MG Management

Abbreviations: AAN, American Academy of Neurology; ABN, Association of British Neurologists; EFNS, European Federation of the Neurological Societies; MG, myasthenia gravis; MGFA, Myasthenia Gravis Foundation of America; NMBC, neuromuscular medicine board certified.

Survey question 3A: "What specific guideline do you use for making treatment decisions for MG patients?" (open-ended question/ unaided response; $n=36$ ); $3 \mathrm{~B}$ : "Which of the following clinical treatment guidelines, if any, is the most useful to you in guiding your clinical management of MG patients? (Select all that apply)”.

$(\mathrm{N}=100)$.

"Other" (verbatim open-text responses from participants) included UpToDate; clinical judgment; neuromuscular society guidelines; lab tests, Tensilon, and nerve stimulation; symptomatic and immunosuppressant; International Consensus Guidance (2016); closely watched pulmonary function tests; safety, need for thymectomy, and minimal immune suppression; weakening neurological system, activities of daily living (ADLs), and new onset of limitations.

* Indicates difference was statistically significant $(P<0.05)$. 


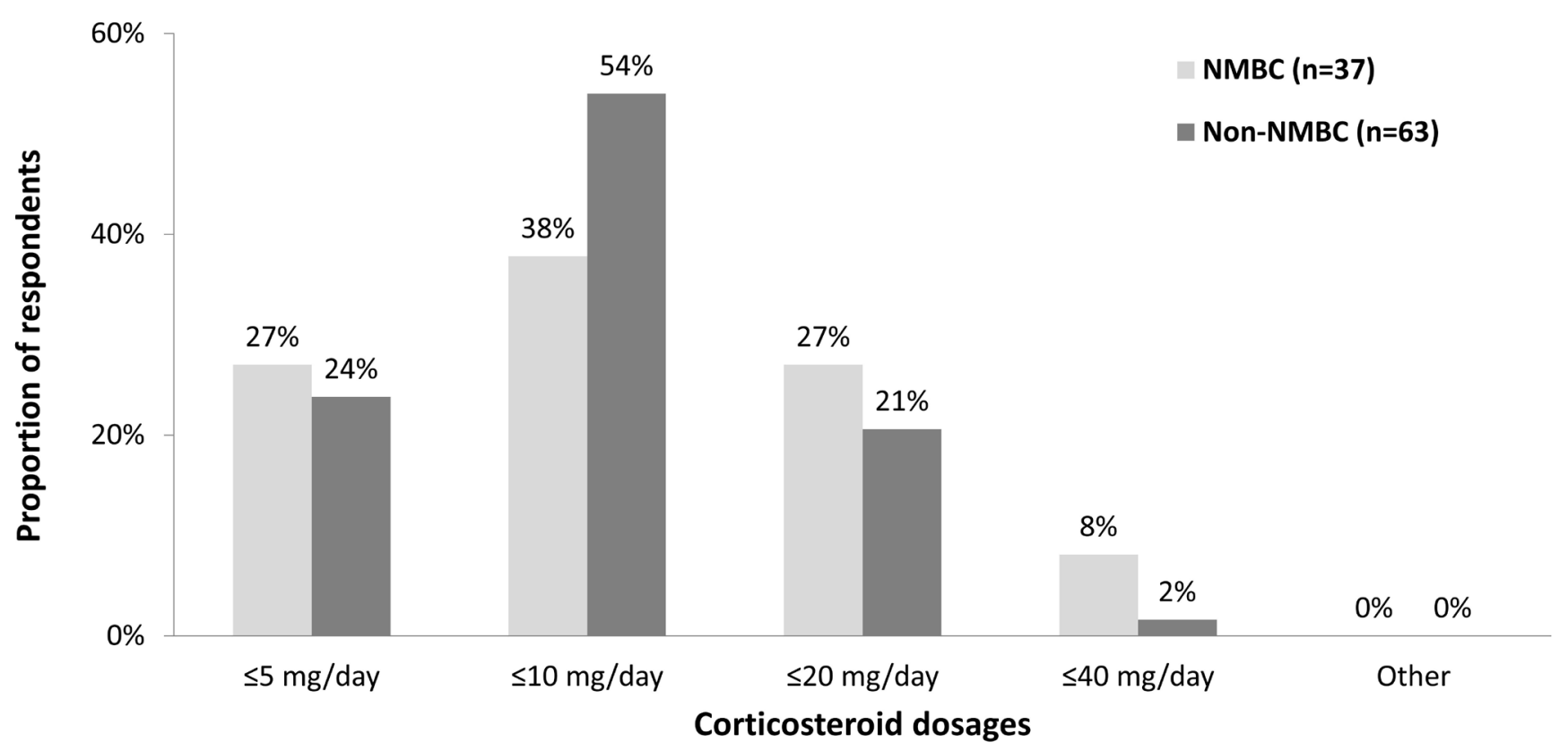

Figure 4: Corticosteroid Dosages Considered Safe

Abbreviations: gMG, generalized myasthenia gravis; NMBC, neuromuscular medicine board certified.

This survey focused on gMG unless otherwise specified.

Survey question: "What chronic, long-term ( $\geq 6$ mo) prednisone-equivalent steroid dose do you consider safe (ie, to minimize adverse events for long-term use)?”.

$\mathrm{N}=100$.

both NMBC and non-NMBC clinicians. Overall, $23 \%$ of respondents said they consider a prednisone-equivalent dose $\leq 20 \mathrm{mg} /$ day safe for $\geq 6$ months' use, with only $4 \%$ reporting doses $\leq 40 \mathrm{mg}$ /day safe for that duration (Figure 4). More NMBC than non-NMBC respondents endorsed higher corticosteroid dosages as safe. To track disease and therapeutic progress, more than $70 \%$ of respondents reported using change in Myasthenia Gravis Activities of Daily Living (MG-ADL) score to help decide when to adjust therapy in a patient with gMG: $30 \%$ reported using a 2-point change, 41\%, a 3-point change. Another $29 \%$ reported not using $\mathrm{MG}-\mathrm{ADL}$ scores for decisions regarding treatment adjustments.

For newly diagnosed patients seropositive for AChR antibodies for whom corticosteroids are contraindicated, responses revealed a preference for IVIg or subcutaneous immunoglobulin (SCIg) over slower-acting NSISTs as the initial treatment choice (Figure 5A). A similar preference was seen with respect to patients with AChR antibody-positive gMG after failure of a first NSIST: $42 \%$ of all respondents said they would use IVIg or SCIg next in such patients rather than another NSIST (Figure 5B). Eculizumab was the next most common treatment. It was selected by $32 \%$ of non-NMBC respondents and $18 \%$ of NMBC respondents, despite its high cost, for patients with AChR antibody-positive gMG in whom a first-choice
NSIST had failed to control symptoms, followed by a second NSIST, rituximab, and TPE. Figure 5C illustrates the various therapeutic approaches used by respondents for newly diagnosed patients with AChR antibody-positive gMG contraindicated for corticosteroids.

Referral practices were also assessed. Statistically significantly more NMBC than non-NMBC respondents (70\% vs 48\%, respectively) reported receiving referrals for a second opinion on diagnosis or management of patients with MG. There was also a statistically significant betweengroup difference for making referrals, with only $11 \%$ of NMBC respondents vs $24 \%$ of non-NMBC respondents referring patients to other providers.

Finally, specific topics respondents said they would like to see targeted in MG treatment guidelines included acute MG crisis management (43\%), followed by initiating/ titrating maintenance medications in chronic MG (29\%) and managing populations of special interest, including pregnancy, pediatric, and ICI-induced MG (23\%). Another $20 \%$ wanted further information on how to treat pure ocular MG, define treatment goals, and track improvement using disease measures. In addition, respondents said they would like to see MG treatment guidelines address management of comorbidities such as diabetes; heart disease; lupus, thyroiditis, and other autoimmune diseases; renal disease; and osteoporosis, in descending order (Figure 6). 
$5 A$

IG
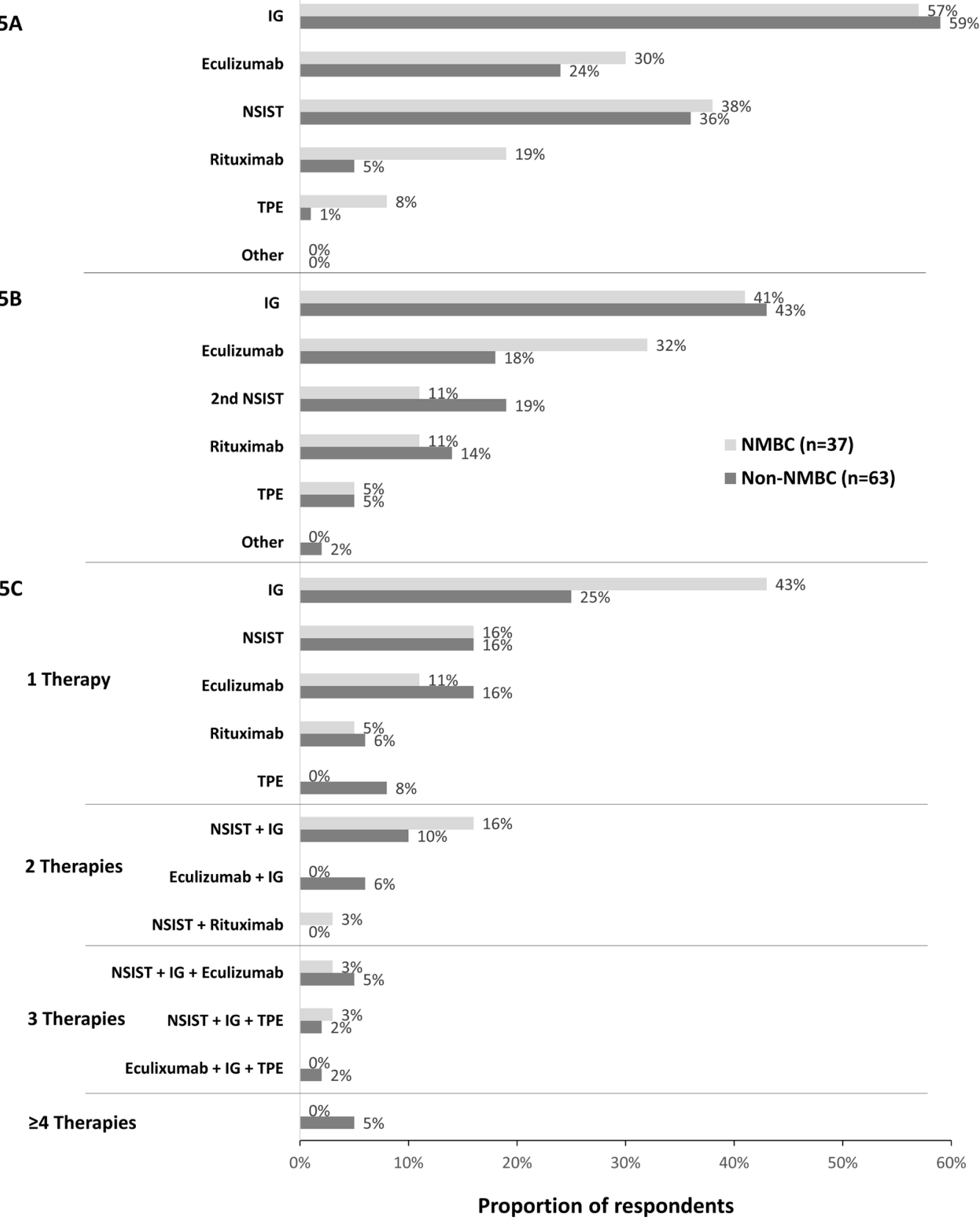

Figure 5. Initial, Second-Line, and Combinatorial Therapeutic Strategies for AChR Antibody-Positive Patients Contraindicated for Steroids

Abbreviations: AChR, acetylcholine receptor; gMG, generalized myasthenia gravis; IG, immunoglobulin (IVIg or SCIg); IVIg, intravenous immunoglobulin; MG-ADL, Myasthenia Gravis Activities of Daily Living; NMBC, neuromuscular medicine board certified; NSIST, nonsteroidal immunosuppressive therapy; SCIg, subcutaneous immunoglobulin; TPE, therapeutic plasma exchange.

Combination therapies were permitted (5C), so percentages may sum $>100 \%$.

Survey question 5A: "In newly diagnosed $\mathrm{AChR}^{+} \mathrm{gMG}$ patients contraindicated for steroids and with moderate functional impairment (MG-ADL >8; moderate severity), what initial therapeutic approach best describes your typical clinical practice?"; 5B: "If the first choice for nonsteroidal immunosuppressant therapy (NSIST) fails to control symptoms, what therapy do you go to next for $\mathrm{AChR}^{+}$patients with gMG?"; 5C: "In newly diagnosed AChR ${ }^{+}$gMG patients contraindicated for steroids and with moderate functional impairment (MG-ADL $>8$; moderate severity), what initial therapeutic approach best describes your typical clinical practice? (Select all agents initiated concurrently)".

$(\mathrm{N}=100)$.

*Indicates difference was statistically significant $(P<0.05)$. 
$6 A$

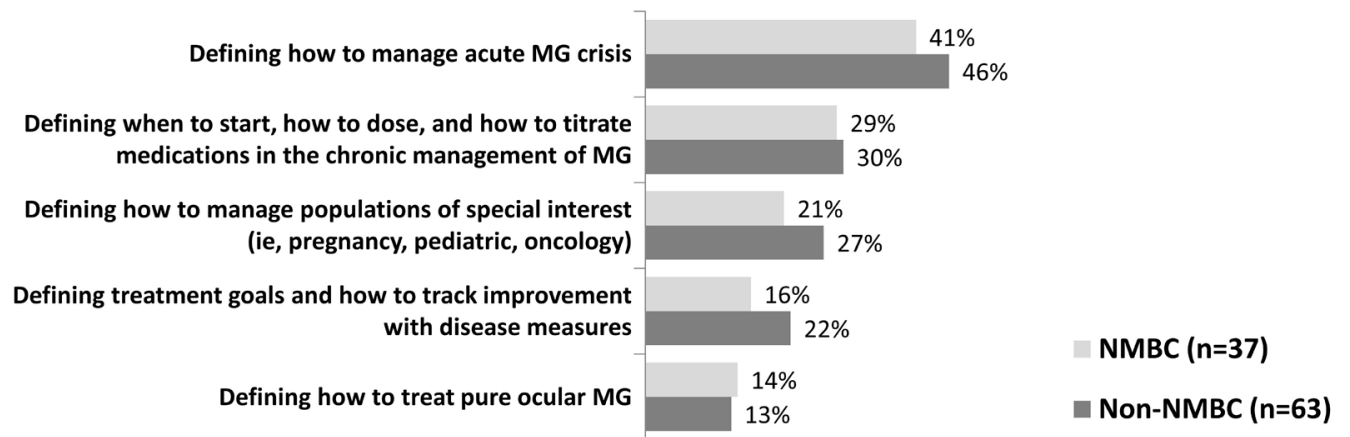

6B

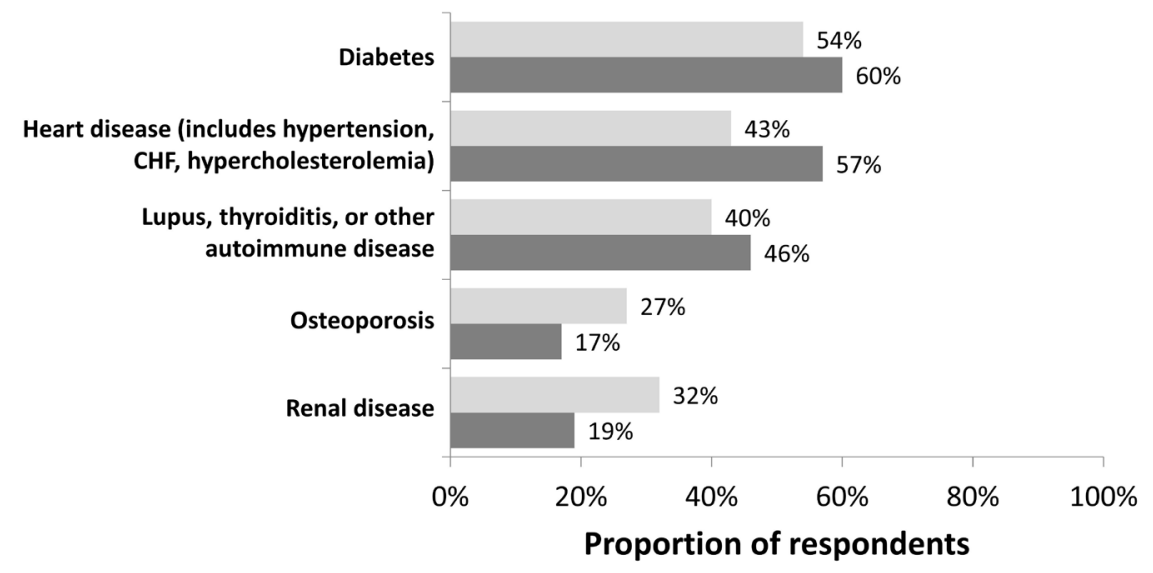

Figure 6. Topics and Comorbidities Chosen as Most Important in Management of MG

Abbreviations: CHF, congestive heart failure; MG, myasthenia gravis; NMBC, neuromuscular medicine board certified.

More than 1 topic could be rated "extremely important," so percentages sum $>100 \%$.

Survey question 6A: "Please rate the following topics in terms of their importance for inclusion in MG treatment guidelines" (5-point Likert scale: not important, minimum importance, important, very important, extremely important); 6B: "Of the comorbidities below, which would you like to see in upcoming guidelines to inform MG treatment decision-making? Please click to indicate the order of importance".

$(\mathrm{N}=100)$.

\section{Discussion}

The relatively low overall percentage of respondents who reported awareness of the 2020 International Consensus Guidance highlights a critical need both for educational outreach to enhance awareness and understanding of guideline recommendations and for their increased dissemination. Awareness was particularly low among non-university-affiliated neurologists, although this is potentially the group for whom the International Consensus Guidance would be most useful. Only $18 \%$ of non-university-affiliated neurologists said they were aware of the 2020 International Consensus Guidance vs $38 \%$ of neurologists who work in university-affiliated settings. It is likely that clinical neurologists working in small practices are faced with difficult treatment decisions but lack the benefit of colleagues who are highly experienced neurologists with whom to discuss challenging cases (14).

The majority of respondents reported not using a specific treatment guideline in making $\mathrm{MG}$ treatment decisions. Of the 36 respondents who did report use of guidelines, $56 \%$ reported using “AAN guidelines," although the AAN itself has not issued specific guidelines for the treatment of MG. The 2016 International Consensus Guidance was published in Neurology, the journal of the AAN, and can be accessed from the AAN website (15). Likewise, the MGFA website has a link to the International Consensus Guidance, which may have driven responses regarding use of "MGFA guidelines"(7).

Despite availability of links to the published consensusbased guidance recommendations on various neurologyassociated websites, aspects of the International Consensus Guidance have not been widely implemented. The 2016 International Consensus Guidance recommended MMS as the goal for treatment of MG (7); however, less than half of the surveyed neurologists use MMS to guide MG treatment decisions. Thirty-seven percent of respondents reported routine use of the Quantitative Myasthenia Gravis (QMG) score to track MG clinically, although this measure requires a spirometer to assess percentage of vital capacity and a dynamometer to assess handgrip strength (17). It is possible that at least some of these respondents do not perform the entire assessment but do utilize some of the strength 
assessments. Similarly, 30\% of respondents reported routinely using Myasthenia Gravis-Manual Muscle Testing (MG-MMT), which is based on routine neurological examination and has the advantage of ease of use.

Managing corticosteroid therapy in MG has been reported as a substantial challenge for some physicians in primary and secondary care settings (2). This ongoing challenge may also indicate a gap in education on, and clinical confidence in, determining a dosage regimen that is high enough to provide clinical benefit but low enough to avoid serious AEs. The majority of neurologists surveyed stated they consider a prednisone-equivalent dose $\leq 10 \mathrm{mg}$ / day safe for $\geq 6$ months' use; only $4 \%$ endorsed doses up to $40 \mathrm{mg}$ per day as safe. Similarly, rheumatology guidelines have defined prednisone-equivalent doses $\leq 5 \mathrm{mg} /$ day as an acceptable maintenance dosage for most patients (16). Guidance on optimal pharmacologic management of MG, including initial and maintenance doses for the most commonly used therapeutics, was included in the supplementary materials of the 2016 International Consensus Guidance (7). The supplementary materials do not seem to have been as well disseminated as the primary document, based on the number of respondents who indicated a need for guidance on this topic.

Cost and availability often factor into clinical treatment decisions. Insurance coverage and formulary issues may have influenced neurologists' reported choices of tests and treatments. Because the range of treatment options for MG is broadening, it is likely that cost will become an even larger factor in treatment decisions (5). Survey results showed that current treatment decision-making often results in a higher cost burden for the patient and health care system: IVIg/SCIg was the most commonly reported second-line therapy for patients with AChR antibodypositive gMG after an NSIST failed to control their symptoms. Surprisingly, IVIg was also the initial therapy of choice, rather than an NSIST, of most respondents to treat newly diagnosed AChR antibody-positive gMG when corticosteroids are contraindicated. Eculizumab, despite its high cost, was the second-most common therapy chosen for such patients. Ease of use and rapidity of onset, compared to other corticosteroid-sparing agents, may be contributory factors to the high reported usage of IVIg and eculizumab.

The International Consensus Guidance recommends referral to a physician or center that specializes in $\mathrm{MG}$ care for patients with refractory MG. A majority of $\mathrm{NMBC}$ respondents reported receiving referrals from other doctors, in accordance with the published guideline. Still, respondents in this survey reported referring only a mean of $19 \%$ of patients with possible MG for a second opinion.

This survey revealed additional topics that should be better addressed, including management of MG populations of special interest, notably ICI-induced MG and ocular MG. Survey respondents requested treatment guidance for rituximab, methotrexate, and eculizumab, all of which were covered in the 2020 update. Survey results also raised educational gaps in management of common comorbidities, including steroid-induced diabetes and cardiac-related issues, as well as lupus, thyroiditis, and other autoimmune diseases.

\section{Limitations}

This study did not address all pertinent issues in the optimal management of patients with MG, and insights and feedback are representative of opinions and practices in the United States only. The focus was primarily on patterns of assessment and therapeutic use of corticosteroids, NSIS'Ts, and autoantibody levels. The survey was designed to determine current levels of knowledge and implementation of existing treatment guidelines. Challenges in managing specific populations or subtypes of MG were not addressed, although they remain important topics for physician education.

\section{Conclusions}

Community neurologists employ various approaches to $\mathrm{MG}$ treatment decision-making, mostly independent of practice guidelines. The majority of respondents were not aware of the updated 2020 International Consensus Guidance; this guidance publication may not be the optimal approach for disseminating to the larger community information regarding consensus-based recommendations for managing $\mathrm{MG}$.

\section{Disclosures}

- This study was funded by argenx, the manufacturer of efgartigimod. Efgartigimod is an investigational agent that is not currently approved for use by any regulatory agency.

- Vera Bril, MD-Ellen and Martin Prosserman Centre for Neuromuscular Diseases, Division of Neurology, Department of Medicine, University Health Network, University of Toronto, Toronto, Ontario, Canada

o Vera Bril has participated on scientific advisory boards of CSL Behring, Baxalta, Grifols, argenx, Octapharma, Alpha Technologies, Powell Mansfield Inc, Shire, Akcea, UCB, and Alnylam. She has received funding for travel or speaker honoraria from CSL Behring and consultancies with CSL Behring, Grifols, Bionevia, Octapharma, Powell Mansfield Inc, argenx, Alpha Technologies, 
Baxalta, Akcea, UCB, Alnylam, and Pfizer.

- Jacqueline Palace, BM, DM-Department of Clinical Neurology, John Radcliffe Hospital, Oxford University Hospitals Trust, Oxford, United Kingdom

o Jacqueline Palace is partly funded by highly specialized services to run a national congenital myasthenia service and a neuromyelitis service. She has received support for scientific meetings and honoraria for advisory work from Merck Serono, Biogen Idec, Novartis, Teva, Chugai Pharma, Bayer Schering, Alexion, Roche, Genzyme, MedImmune, EuroImmun, MedDay, Abide, argenx, UCB, and Viela Bio and grants from Merck Serono, Novartis, Biogen Idec, Teva, Abide, MedImmune, Bayer Schering, Genzyme, Chugai, and Alexion. She has received grants from the Multiple Sclerosis Society UK, Guthy-Jackson Charitable Foundation, National Institute for Health Research, Oxfordshire Health Services Research Committee, Medical Research Council, GMSI (Grant for Multiple Sclerosis Innovation), John Fell Fund, Myaware, and Amplo for research studies.

- Tahseen Mozaffar, MD-UC Irvine-MDA ALS and Neuromuscular Center, University of California, Irvine, California, USA

- Tahseen Mozaffar has served on advisory boards for AbbVie, Alexion, Amicus, argenx, Audentes, Sanofi-Genzyme, Sarepta, and Spark Therapeutics. In relation to these activities, he has received travel reimbursement and honoraria. He has also served on speakers bureaus for Alexion, CSL, Grifols, and Sanofi-Genzyme. He serves on the medical advisory board for The Myositis Association, Neuromuscular Disease Foundation, Myasthenia Gravis Foundation of California, and Myasthenia Gravis Foundation of America. He has received travel funding from The Myositis Association and the Neuromuscular Disease Foundation. He has received research funding from The Myositis Association, the Muscular Dystrophy Association, and from Alexion, Amicus, argenx, Audentes, Bristol Myers Squibb, Cartesian Therapeutics, Grifols, Momenta, Ra Pharmaceuticals, Sanofi-Genzyme, Spark Therapeutics, UCB, and Valerion. He serves on the data safety monitoring board for Acceleron.

- Deborah Gelinas, MD-Medical Affairs, argenx, Boston, Massachusetts, USA

- Edward Brauer, PharmD-Medical Affairs, argenx, Boston, Massachusetts, USA

- Paul Nisbet, PhD-One Research, Mount Pleasant, South Carolina, USA o Paul Nisbet is an employee of One Research. One Research received payment for the conduct of this study and for initial data analysis. He was not compensated for development of this publication.

- Gil I. Wolfe, MD - Department of Neurology, University at Buffalo School of Medicine and Biomedical Sciences, State University of New York, Buffalo, New York, USA

o Gil Wolfe has participated on scientific advisory boards for Grifols, Baxalta, argenx, and UCB and has received speaking honoraria from Grifols and Baxalta.

\section{Acknowledgements}

The authors thank Tam M. Nguyen-Cao, PhD, CMPP, and Susan A. Leon, PhD, of Claritas Scientific LLC for medical writing services and Ann D. Bledsoe Bollert, MA, CMPP, of Y-Axis Editorial for editorial services on this manuscript. Medical writing and editorial support were funded by argenx.

\section{References}

1. Gilhus NE. Myasthenia gravis. N Engl J Med. 2016;375(26):2570-81. Epub 2016/12/29. doi: 10.1056/ NEJMral602678. PubMed PMID: 28029925.

2. Farrugia ME, Goodfellow JA. A practical approach to managing patients with myasthenia gravis-opinions and a review of the literature. Front Neurol. 2020;11:604. Epub 2020/08/01.doi:10.3389/fneur.2020.00604.PubMedPMID: 32733360 ; PubMed Central PMCID: PMCPMC7358547.

3. Lazaridis K, Tzartos SJ. Autoantibody specificities in myasthenia gravis; implications for improved diagnostics and therapeutics. Front Immunol. 2020;11:212. Epub 2020/03/03. doi: 10.3389/fimmu.2020.00212. PubMed PMID: 32117321; PubMed Central PMCID: PMCPMC7033452.

4. Deenen JC, Horlings CG, Verschuuren JJ, Verbeek AL, van Engelen BG. The epidemiology of neuromuscular disorders: A comprehensive overview of the literature. J Neuromuscul Dis. 2015;2(1):73-85. Epub 2015/01/01. PubMed PMID: 28198707.

5. Menon D, Barnett C, Bril V. Novel treatments in myasthenia gravis. Front Neurol. 2020;11:538. Epub 2020/07/28. doi:10.3389/fneur.2020.00538. PubMed PMID: 32714266; PubMed Central PMCID: PMCPMC7344308.

6. Gotterer L, Li Y. Maintenance immunosuppression in myasthenia gravis. J Neurol Sci. 2016;369:294-302. Epub 2016/09/23. doi: 10.1016/j.jns.2016.08.057. PubMed PMID: 27653912.

7. Sanders DB, Wolfe GI, Benatar M, Evoli A, Gilhus NE, Illa I, et al. International Consensus Guidance for Management of Myasthenia Gravis: executive summary. 
Neurology.2016;87(4):419-25. Epub 2016/07/01. doi:10.1212/ WNL.0000000000002790. PubMed PMID: 27358333; PubMed Central PMCID: PMCPMC4977114.

8. Narayanaswami P, Sanders DB, Wolfe G, Benatar M, Cea G, Evoli A, et al. International Consensus Guidance for Management of Myasthenia Gravis: 2020 update. Neurology. 2021;96(3):114-22. Epub 2020/11/05. doi: 10.1212/ WNL.0000000000011124. PubMed PMID: 33144515; PubMed Central PMCID: PMCPMC7884987.

9. Gable KL, Guptill JT. Antagonism of the neonatal Fc receptor as an emerging treatment for myasthenia gravis. Front Immunol. 2019;10:3052. Epub 2020/01/31. doi: 10.3389/ fimmu.2019.03052. PubMed PMID: 31998320; PubMed Central PMCID: PMCPMC6965493.

10. Farmakidis C, Pasnoor M, Dimachkie MM, Barohn RJ. Treatment of myasthenia gravis. Neurol Clin. 2018;36(2):311-37. Epub 2018/04/16. doi: 10.1016/j. ncl.2018.01.011. PubMed PMID: 29655452; PubMed Central PMCID: PMCPMC6690491.

11. Dalakas MC. Progress in the therapy of myasthenia gravis: getting closer to effective targeted immunotherapies. Curr Opin Neurol. 2020;33(5):545-52. Epub 2020/08/25. doi: 10.1097/WCO.0000000000000858. PubMed PMID: 32833750 .

12. Mantegazza R, Antozzi C. When myasthenia gravis is deemed refractory: clinical signposts and treatment strategies. Ther Adv Neurol Disord. 2018;11:1756285617749134. Epub
2018/02/07.doi:10.1177/1756285617749134.PubMed PMID: 29403543; PubMed Central PMCID: PMCPMC5791553.

13. Howard JF, Jr., Bril V, Vu T, Karam C, Peric $\mathrm{S}$, Margania T, et al. Safety, efficacy, and tolerability of efgartigimod in patients with generalised myasthenia gravis (ADAPT): a multicentre, randomised, placebo-controlled, phase 3 trial. Lancet Neurol. 2021;20(7):526-36. Epub 2021/06/20. doi: 10.1016/S1474-4422(21)00159-9. PubMed PMID: 34146511.

14. Jayalakshmi S, Vooturi S. Legal challenges in neurological practice. Ann Indian Acad Neurol. 2016;19(Suppl 1):S3-S8. Epub 2016/11/29. doi: 10.4103/0972-2327.192888. PubMed PMID: 27891018; PubMed Central PMCID: PMCPMC5109758.

15. American Academy of Neurology. Policy \& Guidelines/Neuromuscular [February 10, 2021]. Available from: https://www.aan.com/Guidelines/home/ ByTopic?topicId=19.

16. Buttgereit F, Bijlsma JW. Glucocorticoids in rheumatoid arthritis: the picture is shaping up. Ann Rheum Dis. 2017;76(11):1785-7. Epub 2017/05/06. doi: 10.1136/ annrheumdis-2017-211187. PubMed PMID: 28473424.

17. Barnett C, Herbelin L, Dimachkie MM, Barohn RJ. Measuring clinical treatment response in myasthenia gravis. Neurol Clin. 2018;36(2):339-353. doi:10.1016/j. ncl.2018.01.006. 


\section{Appendix A: Survey Measure}

\section{MG Guidelines Study Quantitative Survey Instrument \\ 10-minute online survey $(\mathrm{N}=100)$}

\section{Main survey \\ Treatment Questions:}

\section{[PREVIOUS Q9]}

1) This survey focuses on generalized myasthenia gravis (MG) unless otherwise specified. Which of the following treatment goal(s) do you use to guide MG treatment decisions?

a. Minimum Symptom Expression (MSE) - MG-ADL Score of O or 1; regardless of taking MG therapy or not.

b. Complete Stable Remission (CSR) - The patient has had no symptoms or signs of MG for at least 1 year and has received no therapy for MG during that time. There is no weakness of any muscle on careful examination by someone skilled in the evaluation of neuromuscular disease. Isolated weakness of eyelid closure is accepted.

c. Pharmacological Remission (PR) - Same as CSR, but the patient is still receiving some form of pharmacologic therapy for MG. Patients taking AChEi are excluded from this category because their use suggests the presence of weakness.

d. Minimal Manifestation Status (MMS) - The patient has no symptoms or functional limitations from MG but has some weakness on examination of some muscles. This class recognizes that some patients who otherwise meet the definition of CSR or PR do have weakness that is only detectable by careful examination.

e. Patient Acceptable Symptom State (PASS) Question - 'Yes' Response as the Goal to the following question: Considering all the ways you are affected by Myasthenia, if you had to stay in your current state for the next few months, would you say that your current disease status is satisfactory?

f. Clinical judgement of patient strength, lifestyle activities, and tolerance to treatment.

g. None of the above [EXCLUSIVE]

\section{[PREVIOUS Q8]}

2) Which disease specific measures do you use routinely in your clinic to track MG status? Select all that apply.

1. Myasthenia Gravis Activities of Daily Living (MG-ADL)

2. Quantitative Myasthenia Gravis Score (QMG)

3. Myasthenia Gravis Composite Score (MGC)

4. Myasthenia Gravis Quality of Life revised 15 (MG-QoL15r)

5. Myasthenia Gravis-Manual Muscle Testing (MG-MMT)

6. Medical Research Council Scale for Muscle Strength (MRC)

7. Patient Acceptable Symptom State (PASS)

8. Other [SPECIFY]

9. None of these [EXCLUSIVE] 
10) Deleted

11) Would you offer thymectomy to patients with purely ocular MG (MGFA Class 1)?

1. Yes

2. No

12) Please indicate whether you offer thymectomy in generalized MG patients with the following antibody status.

\section{Yes No}

1. $\mathrm{AChR}^{+}$

2. $\mathrm{MuSK}^{+}$

3. LRP4

4. Agrin

5. Seronegative

\begin{tabular}{|l|l|}
\hline & \\
\hline & \\
\hline & \\
\hline & \\
\hline & \\
\hline
\end{tabular}

13) With what percent of your $\mathrm{AChR} \mathrm{Ab}$ patients do you discuss thymectomy?

[RANGE O - 100]

$\left.\left.\right|_{-}\right|_{-}|-|$percent of $\mathrm{AChR} \mathrm{Ab}^{+}$patients under 65 with whom you discuss thymectomy

14) What percentage of your $\mathrm{AChR} \mathrm{Ab}$ patients undergo thymectomy?

[RANGE 0 - 100]

$\left.\left.\left.\right|_{-}\right|_{-}\right|_{-}$percent of $\mathrm{AChR} \mathrm{Ab^{+ }}$ patients under 65 undergo thymectomy

15) DELETED

[IF ANY PATIENTS UNDERGO THYMECTOMY (Q14>1), ASK QUESTION]

16) Among your patients who undergo thymectomy, what percent undergo each of the following procedures?

[RANGE:0-100]

1. Minimally invasive $\left.\left.\left.\quad\right|_{-}\right|_{-}\right|_{-} \mid$percent

2. Trans-sternal $\left.\left.\right|_{-}\right|_{-}|-|$percent

[SHOW RUNNING TOTAL; MUST SUM TO 100\%]

17) When initiating steroids on an outpatient basis in a newly diagnosed generalized MG patient, what prednisone equivalent dose do you start?

[RANGE: 0-100.00]

$\left.\left.\left.\right|_{-}\right|_{-}\right|_{-} \mid \mathrm{mg} / \mathrm{d}$

18) What is the maximum steroid dose you would use to achieve disease control?

[RANGE: 0-100.00]

${ }_{-}|+|-\mid \mathrm{mg} / \mathrm{kg} / \mathrm{d}$

19) Do you initiate with daily steroid dosing or every-other-day steroid dosing?

1. Daily (QD)

2. Every Other Day (QOD) 
20) Upon initiation of steroids, on average, how long does it generally take to reach a minimally effective dose and then begin tapering steroids?

1. 1 month

2. 3 months

3. 6 months

4. 12 months

5. 18 months

6. 24 months

7. $>24$ months

21) What chronic, long-term ( $\geq 6 \mathrm{mo}$ ) prednisone-equivalent steroid dose do you consider safe (ie, to minimize AEs for long-term use)?
a. $\quad \leq 5 \mathrm{mg}$ per day
b. $\quad \leq 10 \mathrm{mg}$ per day
c. $\leq 20 \mathrm{mg}$ per day
d. $\leq 40 \mathrm{mg}$ per day
e. Other

22) When assessing a generalized MG patient, what change in MG-ADL score would cause you to make a change in the patient's MG treatment?
a. 2-point change in $\mathrm{MG}$-ADL
b. 3-point change in MG-ADL
c. I do not make treatment decisions based on MG-ADL

23) In general, from the time of starting a steroid, how long do you wait for a clinical response to steroids before determining the need to add a nonsteroidal immunosuppressant therapy (NSIST)?
a. I do not wait; I start an NSIST and steroid at the same time
b. 1 month
c. 3 months
d. 6 months
e. $>6$ months

23b) How long does it take for you to begin to wean?
a. 1 month
b. 3 months
c. 6 months
d. >6 months 
24) Which nonsteroidal below do you prefer as lst line after steroid initiation? Select one response only.

[RANDOMIZE]
a. Azathioprine (Azasan ${ }^{\oplus}$, Imuran $\left.{ }^{\oplus}\right)$
b. Cyclosporine $\left(\right.$ Gengraf $^{\oplus}$, Neoral ${ }^{\circledast}$, Sandimmune $\left.{ }^{\circledast}\right)$
c. Mycophenolate (CellCept ${ }^{\oplus}$, Myfortic $\left.^{\varpi}\right)$
d. Methotrexate (Rheumatrex ${ }^{\oplus}$, Trexall ${ }^{\star}$ )
e. Tacrolimus (Astagraf XL ${ }^{\oplus}$, Envarsus XR ${ }^{\oplus}$, Prograf $^{\star}$ )
f. Cyclophosphamide (Cytoxan $\left.{ }^{\circledR}\right)$
g. Other [SPECIFY]

25) Please briefly describe why [NSIST FROM Q24] is your lst line preferred NSIST after steroid initiation.

26) With what [NSIST FROM Q24] dose do you initiate as a starting dose? [RANGE: 0-999]

[IF Q24=A (Azathioprine), SHOW OPTIONS]

$\left.\left.\left.\right|_{-}\right|_{-}\right|_{-}$mg/day by mouth

[IF Q24=B (Cyclosporine), SHOW OPTIONS]

$\left.\left.\right|_{-}\right|_{-}|+| \mathrm{mg} / \mathrm{kg} /$ day by mouth

[IF Q24=C (Mycophenolate), SHOW OPTIONS. ALLOW ONE OPTION TO BE BLANK.]

$\left.\left.\left.\right|_{-}\right|_{-}\right|_{-} \mathrm{mg} /$ day by mouth

$\left.\left.\left.\right|_{-}\right|_{-}\right|_{-} \mathrm{mg}$ /day by IV infusion

[IF Q24=D (Methotrexate), SHOW OPTIONS]

$\left.\left.\left.\right|_{-}\right|_{-}\right|_{-} \mid \mathrm{mg} /$ week

[IF Q24=E (Tacrolimus), SHOW OPTIONS]

$\left.\left.\left.\right|_{-}\right|_{-}\right|_{-} \mid \mathrm{mg} / \mathrm{kg} /$ day by mouth

[IF Q24=F (Cyclophosphamide), SHOW OPTIONS. ALLOW ONE OPTION TO BE BLANK]

$\left.\left.\right|_{-}\right|_{-}|+| \mathrm{mg} / \mathrm{kg} /$ day by mouth

$\left.\left.\right|_{-}\right|_{-}|+| \mathrm{mg} / \mathrm{kg} /$ day by IV infusion

[IF Q24=G (Other), SHOW OPTIONS.]

[TEXT BOX]

27) How high of a/an [INSERT NSID FROM Q24] dose do you use? [RANGE: 0-9999] [IF Q24=A (Azathioprine), SHOW OPTIONS]

$\left.\left.\right|_{-}{ }_{-}\right|_{-} \mid \mathrm{mg} /$ day by mouth

[IF Q24=B (Cyclosporine), SHOW OPTIONS]

$\left.\left.\right|_{-}\right|_{-}{ }_{-} \mid \mathrm{mg} / \mathrm{kg} /$ day by mouth 
[IF Q24=C (Mycophenolate), SHOW OPTIONS. ALLOW ONE OPTION TO BE BLANK]

$\left.\left.\left.\right|_{-}\right|_{-}\right|_{-} \mid \mathrm{mg} /$ day by mouth

$\left.\left.\right|_{-}\right|_{-}|-| \mathrm{mg} /$ day by IV infusion

[IF Q24=D (Methotrexate), SHOW OPTIONS]

$\left.\right|_{-}{ }_{-}|-| \mathrm{mg} /$ week

[IF Q24=E (Tacrolimus), SHOW OPTIONS]

$\left.\left.\left.\right|_{-}\right|_{-}\right|_{-} \mathrm{mg} / \mathrm{kg} /$ day by mouth

[IF Q24=F (Cyclophosphamide), SHOW OPTIONS. ALLOW ONE OPTION TO BE BLANK]

$\left.\left.\right|_{-}\right|_{-}{ }_{-} \mid \mathrm{mg} / \mathrm{kg} /$ day by mouth

$\left.\left.\right|_{-}\right|_{-}|-| \mathrm{mg} / \mathrm{kg} /$ day by IV infusion

[IF Q24=G (Other), SHOW OPTIONS.]

[TEXT BOX]

28) What duration of [INSERT NSIST FROM Q24], do you allow before determining lack of efficacy?
a. 1 month
b. 3 months
c. 6 months
d. 6-12 months
e. $12-18$ months
f. $>18$ months

29) In a typical MG patient, if symptoms are controlled, how long do you typically wait before attempting to taper nonsteroidal immunosuppressants (NSIST) after disease control is attained?
a. 0 to 3 months
b. 4 to 6 months
c. 6 to 12 months
d. 12 to 24 months
e. $>24$ months
f. I do not taper

30) If the lst choice for nonsteroidal immunosuppressant therapy (NSIST) fails to control symptoms, what therapy do you go to next for $\mathrm{AChR}-\mathrm{Ab}^{+}$patients with generalized $\mathrm{MG}$ ?
a. A second nonsteroidal immunosuppressive therapy (NSIST)
b. Immune Globulin (IVIg or SCIg)
c. Eculizumab
d. Rituximab
e. Therapeutic Plasma Exchange
f. Other 
31) In newly diagnosed $\mathrm{AChR}-\mathrm{Ab}^{+}$generalized $\mathrm{MG}$ patients contraindicated for steroids, and with moderate functional impairment (MG-ADL > 8; moderate severity), what initial therapeutic approach best describes your typical clinical practice? Please select multiple agents ONLY if you initiate more than one agent at the same time. [MULTIPLE RESPONSE]
a. A nonsteroidal immunosuppressive therapy (NSIST)
b. Immune Globulin (IVIg or SCIg)
c. Eculizumab
d. Rituximab
e. Therapeutic Plasma Exchange
f. Other

32) In what percentage of generalized MG patients do you avoid steroids?

[RANGE 0 - 100]

$\left.\left.\right|_{-}\right|_{-}|-|$percent

33) Which clinical labs do you routinely monitor in an MG patient? Please specify any additional monitoring for your choice of nonsteroidal immunosuppressant therapy (NSIST)?

34) Do you monitor autoantibody levels in an MG patient to evaluate response to therapy?
a. Yes
b. No

[IF Q34=1 (Yes), ASK Q35]

35) Which of the following autoantibody levels do you use to evaluate response of an MG patient to therapy? Check all that apply.
a. None $[\mathrm{ANCHOR}]$
b. $\mathrm{AChR}^{+}$
c. MuSK
d. LRP4
e. Agrin
f. Other [TEXT BOX]

36) Which of the following treatments would you consider using in treating a MuSK+ patient? Check all that apply.
a. AChEi
b. Steroids
c. Rituximab
d. NSIST
e. IVIg and/or SCIg
f. Eculizumab
g. Plasma exchange 
37) Do you recommend vaccine boosters to your MG patients?
a. Yes
b. No
c. Conditional recommendation; please specify [SPECIFY]

38) Which vaccines do you typically recommend? Select all that apply.
a. Pneumococcal
b. Influenza
c. Varicella zoster
d. Tetanus
e. Meningococcal
f. Other [SPECIFY]
g. None, due to concern of exacerbating MG [EXCLUSIVE]

39) What is your preferred treatment for managing MG crisis?
a. IVIg
b. PLEX
c. High-dose steroids
d. Other

\section{General Guideline Questions:}

40) When you are making a treatment decision for a patient diagnosed with myasthenia gravis (MG) do you use specific clinical treatment guidelines to make treatment decisions for your patients?

a. Yes

b. No, I do not use a specific guideline

\section{[ASK Q41 IF USE SPECIFIC GUIDELINE (Q40=YES)]}

41) What specific guideline do you use for making treatment decisions for MG patients?

42) Which of the following clinical treatment guidelines, if any, is the most useful to you in guiding your clinical management of MG patients? Select all that apply.

[RANDOMIZE]

a. Association of British Neurology (ABN) Myasthenia Gravis Management Guidelines

b. European Federation of Neurological Societies (EFNS) Guidelines for the Treatment of Autoimmune Neuromuscular Transmission Disorders

c. International Consensus Guidance for the management of myasthenia gravis (MG Foundation of America [MGFA]-appointed Task Force guidelines)

d. UpToDate ${ }^{\circledast}$ (Wolters Kluwer UpToDate, Inc.)

e. Other [SPECIFY] [ANCHOR]

f. I do not use a specific guideline 
43) Please rate the following topics in terms of their importance for inclusion in MG treatment guidelines.

\begin{tabular}{|c|c|c|c|c|c|}
\hline [RANDOMIZE] & $\begin{array}{c}\text { Not } \\
\text { important }\end{array}$ & $\begin{array}{l}\text { Minimum } \\
\text { importance }\end{array}$ & Important & $\begin{array}{c}\text { Very } \\
\text { important }\end{array}$ & $\begin{array}{l}\text { Extremely } \\
\text { important }\end{array}$ \\
\hline $\begin{array}{l}\text { Defining treatment goals an } \\
\text { how to track improvement } \\
\text { with disease measures }\end{array}$ & & & & & \\
\hline $\begin{array}{l}\text { Defining when to start, how } \\
\text { to dose and how to titrate } \\
\text { medications in the chronic } \\
\text { management of MG }\end{array}$ & & & & & \\
\hline $\begin{array}{l}\text { Defining how to manage } \\
\text { populations of special inter } \\
\text { (i.e., pregnancy, children, } \\
\text { cancer patients) }\end{array}$ & & & & & \\
\hline $\begin{array}{l}\text { Defining how to manage act } \\
\text { MG crisis }\end{array}$ & & & & & \\
\hline $\begin{array}{l}\text { Defining how to treat pure } \\
\text { ocular patients }\end{array}$ & & & & & \\
\hline
\end{tabular}

44) Of the comorbidities below, which would you like to see in upcoming guidelines to inform MG treatment decision making? Please click to indicate the order of importance.

[SORTING TASK]
a. Diabetes
b. Lupus, thyroiditis or other autoimmune disease
c. Heart disease (including hypertension, CHF, hypercholesterolemia)
d. Osteoporosis
e. Renal disease
f. Other [SPECIFY]

45) Which diagnostic test(s) do you regularly perform to confirm MG? Select all that apply.
a. AChEi challenge
b. Repetitive nerve stimulation
c. Single-fiber EMG
d. AChR antibody panel
e. MuSK antibody
f. LRP4 antibody
g. Ice pack test
h. Other [SPECIFY]

46) How do you confirm MG diagnosis in a seronegative patient?

[ALLOW MULTIPLE RESPONSE]
a. AChEi challenge
b. Repetitive nerve stimulation
c. Single-fiber EMG
d. Other [SPECIFY] 
47) Do other neurologists refer patients to you for a second opinion on the diagnosis/management of MG?
a. Yes
b. No

48) What percentage of possible MG patients do you refer for a second opinion? [RANGE: 0-100]

$\left.\left.\left.\right|_{-}\right|_{-}\right|_{-}$percent of possible MG patients referred for a second opinion

49) Are you currently aware of the newly released 2020 International Consensus Guidelines developed with conjunction with the MGFA Task Force?
a. Yes
b. No

50) Do you use rituximab in $\mathrm{AChR}^{+} \mathrm{MG}$ patients?
a. Yes
b. No

51) Do you use eculizumab in non-refractory MG patients?
a. Yes
b. No

52) Do you use eculizumab in seronegative MG patients?
a. Yes
b. No

53) Have you treated patients with immune checkpoint inhibitor-induced MG?
a. Yes
b. No

54) What is your treatment of choice for immune checkpoint inhibitor-induced MG? Select all that apply.
a. Steroids
b. Plasma exchange
c. IVIg
d. Other [SPECIFY]

[END OF SURVEY] 
Appendix B. Treatment goals as defined in survey

\begin{tabular}{|l|l|}
\hline $\begin{array}{l}\text { Minimum symptom } \\
\text { expression (MSE) }\end{array}$ & MG-ADL score of O or l, regardless whether taking MG therapy or not. \\
\hline $\begin{array}{l}\text { Complete stable } \\
\text { remission (CSR) }\end{array}$ & $\begin{array}{l}\text { Patient has had no symptoms or signs of MG for } \leq 1 \text { year, with no therapy for MG } \\
\text { during that time. There is no weakness of any muscle on careful examination by } \\
\text { someone skilled in the evaluation of neuromuscular disease. Isolated weakness } \\
\text { of eyelid closure is accepted. }\end{array}$ \\
\hline $\begin{array}{l}\text { Pharmacologic } \\
\text { remission (PR) }\end{array}$ & $\begin{array}{l}\text { Same as CSR, but the patient is still receiving some form of pharmacologic } \\
\text { therapy for MG. Patients taking AChEi are excluded from this category because } \\
\text { their use suggests the presence of weakness. }\end{array}$ \\
\hline $\begin{array}{l}\text { Minimal } \\
\text { manifestation status } \\
\text { (MMS) }\end{array}$ & $\begin{array}{l}\text { The patient has no symptoms or functional limitation from MG but has some } \\
\text { weakness on examination of some muscles. This class recognizes that some } \\
\text { patients who otherwise meet the definition of CSR or PR do have weakness that } \\
\text { is only detectable by careful examination. }\end{array}$ \\
\hline $\begin{array}{l}\text { Patient Acceptable } \\
\text { Symptom State } \\
\text { (PASS) question }\end{array}$ & $\begin{array}{l}\text { Positive (ie, "yes") response to the following question: Considering all the ways } \\
\text { you are affected by MG, if you had to stay in your current state for the next few } \\
\text { months, would you say that your current disease status is satisfactory? }\end{array}$ \\
\hline Clinical judgment & Judgment of patient strength, ADLs, and tolerance to treatment. \\
\hline
\end{tabular}

Abbreviations: AChEi, acetylcholinesterase inhibitor; ADL, activity of daily living; MG, myasthenia gravis; MG-ADL, Myasthenia Gravis Activities of Daily Living. 